\title{
REVIEW OF THERAPEUTICS
}

\section{Pleconaril, a Novel Antipicornaviral Agent}

\author{
Naomi R. Florea, Pharm.D., Dana Maglio, Pharm.D., and David P. Nicolau, Pharm.D., FCCP \\ Despite the availability of therapy for selected symptoms, no specific antiviral \\ agents are available to treat or prevent infections due to the viruses of the \\ Picornaviridae family - rhinoviruses and enteroviruses. Characterization of \\ the three-dimensional structure of picornaviruses in the 1980s allowed \\ development of compounds targeted at the virus itself. Pleconaril is a novel, \\ orally available, systemically acting molecule whose pharmacokinetics are \\ characterized by a two-compartment open model with first-order absorption \\ and with a safety profile similar to that of placebo. It shows promising results \\ in treatment of picornaviral respiratory tract infections, meningitis, and other \\ life-threatening infections.
}

(Pharmacotherapy 2003;23(3):339-348)

\section{OUTLINE}

\section{Rhinoviruses \\ Enteroviruses \\ Treatment \\ Pleconaril \\ Pharmacology \\ Pharmacokinetics \\ Clinical Efficacy and Safety \\ Place in Therapy}

The most common viruses implicated in respiratory infections, and among the most prevalent and clinically important viral pathogens infecting humans, are those of the Picornaviridae family-rhinoviruses and enteroviruses. In the United States alone there are over 1 billion colds annually, ${ }^{1}$ of which rhinoviruses are the leading cause. Certain patients are at risk for complications due to the common cold, such as those with asthma, chronic heart failure, bronchiectasis, and cystic fibrosis. Rhinoviruses are also a predominant cause of asthma exacerbations in adults and children ${ }^{2,3}$ and severe lower respiratory tract disease, including acute exacerbations of chronic bronchitis. Enteroviruses are the most common

From the Department of Pharmacy Research (all authors) and the Division of Infectious Diseases (Dr. Nicolau), Hartford Hospital, Hartford, Connecticut.

Address reprint requests to David P. Nicolau, Pharm.D., Department of Pharmacy Research, Hartford Hospital, 80 Seymour Street, Hartford, CT 06102; e-mail: dnicola@harthosp.org. cause of viral meningitis in the United States and an important cause of encephalitis, poliomyelitis, myocarditis, hemorrhagic conjunctivitis, handfoot-mouth syndrome, pleurodynia, and nonspecific febrile illnesses. ${ }^{4,5}$ They cause approximately 10-15 million infections annually. ${ }^{6}$ They are important pathogens in central nervous system (CNS) infections and target a variety of other organ systems as well.

Treatment for these viral infections is limited to symptomatic remedies. Elucidation of the structure of picornaviruses in 1985 provided the means to identify a potential target for specific antiviral pharmacologic therapy. Pleconaril is the most advanced antipicornaviral compound developed thus far and is being studied in clinical trials for treatment of various infectious diseases due to rhinoviruses and enteroviruses.

\section{Rhinoviruses}

Viral respiratory infections have several etiologies (Table 1). ${ }^{7}$ During seasonal epidemics, rhinoviruses, with more than 100 serotypes, are implicated in $50-80 \%$ of cases of the common cold. ${ }^{8}$ Other viruses associated with the common cold are coronaviruses, respiratory syncytial virus, influenza virus, parainfluenza virus, and adenovirus. They can be distinguished from rhinoviruses in that they cause a much smaller proportion of infections, or cause a higher proportion of lower respiratory or systemic 
Table 1. Etiologies of Viral Respiratory Infections ${ }^{7}$

\begin{tabular}{lc}
\hline Pathogen & $\begin{array}{c}\text { Frequency of } \\
\text { Infection (\%) }\end{array}$ \\
\hline Rhinoviruses (> 100 serotypes) & 40 \\
Enteroviruses & $5-15$ \\
Coronaviruses & $5-15$ \\
Respiratory syncytial virus & $5-15$ \\
Influenza virus & $1-5$ \\
Parainfluenza virus & $1-5$ \\
\hline
\end{tabular}

symptoms in addition to nasal symptoms characteristic of colds. With the exception of parainfluenza virus, rhinoviruses can further be distinguished from other respiratory viruses by their ability to cause infection throughout the year, with marked peaks in spring and early fall in temperate regions. The epidemiology of other respiratory viruses is characterized by distinct seasonal peaks, with minimal frequency of infection between peaks. ${ }^{9}$

Rhinovirus contains four structural proteinsVP1, VP2, VP3, and VP4- that form a nonenveloped, icosahedral capsid. Deep canyons on the capsid surface contain a receptor-binding domain. The virus evades the host immune system because the capsid surface mutates constantly, although the canyons maintain their antigenic specificity. The canyons are too narrow to accommodate the Fab antibody portion, thereby escaping host deactivation, but are large enough to bind the host cell receptor. The intercellular adhesion receptor molecule- 1 (ICAM-1) is the major cellular receptor for $90 \%$ of rhinoviruses. This receptor is expressed on nasal epithelial cells, with high concentrations in adenoid sinuses. ${ }^{10}$

The pathogenesis of rhinovirus infections is not completely understood. Most exposures to the virus result in infection in the absence of specific immunity to the infecting serotype. Transmission occurs to some extent through inhalation of aerosolized particles but mostly through hand-nose or hand-eye contact after contamination of the hand with infected secretions. Rhinovirus then invades the host by binding to its cellular receptor, ICAM-1, on nasal epithelial cells. After host cell invasion and replication, the rhinovirus spreads to epithelial cells of the nasal passages to the pharynx and, in some people, may spread to the large airways. ${ }^{11}$

Symptoms of the common cold are generally mild to moderate in healthy individuals. They usually begin 1-2 days after infection, with peak symptoms occurring after 2-4 days. Early symptoms include nasal obstruction, rhinorrhea, sneezing, and sore or scratchy throat. Cough is present in approximately $30 \%$ of colds and is reported to be the most bothersome and persistent symptom later in the disease course. ${ }^{12}$ Most rhinovirus colds last 1 week, but approximately $25 \%$ last 2 weeks. ${ }^{2}$

In addition to mildly symptomatic colds, rhinovirus infections are associated with a number of upper and lower respiratory tract complications that can have a significant medical impact. Respiratory viruses are important predisposing factors in the development of acute bacterial otitis media, particularly in children. The local edema associated with viral respiratory infections presumably causes obstruction of the eustachian tube, which can lead to the development of acute bacterial otitis media. Rhinovirus has been reported as the most common virus associated with otitis media. Similarly, rhinoviral infection may predispose patients to bacterial sinusitis by entrapping bacteria in the sinus cavity. ${ }^{13}$

Rhinoviruses are increasingly associated with lower respiratory tract illness in adults and children. A study in adults aged $60-90$ years reported lower respiratory tract symptoms in $65 \%$ of rhinoviral infections. Of those who consulted a doctor, $75 \%$ received antibiotic treatment. ${ }^{14}$ Evaluation of an outbreak of rhinovirus infection in a long-term care facility demonstrated the capability of the virus to cause severe respiratory illness, especially in patients with underlying lung disease. ${ }^{15}$ In infants less than 12 months of age, infection required hospitalization for lower respiratory tract symptoms, especially bronchiolitis, and deterioration in bronchopulmonary dysplasia. ${ }^{16,17}$ Rhinoviruses were implicated in exacerbations of asthma in adults and children. ${ }^{18,19}$ In patients older than 2 years, they were the most frequently isolated pathogens in asthma exacerbations and hospitalizations. ${ }^{20}$ In addition, asthmatic children experience more frequent rhinovirus infections than their siblings, and the infections last longer. ${ }^{21}$ Rhinoviruses also are implicated in up to $40 \%$ of exacerbations of chronic bronchitis. $^{22}$

\section{Enteroviruses}

Enteroviruses have more than 65 serotypes. They are classified into five groups: polioviruses, group A coxsackieviruses, group B coxsackieviruses, echoviruses, and numbered enteroviruses. 
Although polio infections are contained in most countries, nonpolio enteroviruses continue to cause disease. Most patients are young infants, but adults can be affected as well. The enterovirus groups share a similar pathogenic process of disease but differ in the target organ affected after blood-borne dissemination. Enteroviral infection results from fecal-oral contamination and to a lesser degree respiratory inhalation. Viral replication can occur in the nasopharynx, but the principal site of viral entry is the gastrointestinal tract.

Enteroviruses are responsible for a wide spectrum of infections involving almost every organ system ranging from nonfocal febrile illness to encephalitis, myocarditis, and fulminant neonatal sepsis. ${ }^{23}$ Infection of the CNS ranges from meningitis, which generally is associated with an excellent prognosis, to encephalitis, which can be associated with profound acute disease and long-term sequelae or even death. Enteroviral infection also commonly causes nonspecific febrile illness that affects 10-15 million people in the United States annually. ${ }^{1}$ The infection may also present as a vesicular disease affecting the skin as in handfoot-mouth disease and herpangina. In addition, patients can present with myocarditis, hemorrhagic conjunctivitis, and pleurodynia.

Most infections are characterized by a mild disease and positive prognosis; however, serious complications can occur and not all patients have a benign disease course. A report of enteroviral infection in infants described progression to fulminant, nearly fatal cardiopulmonary disease. ${ }^{24}$ Some strains caused acute fatal epidemics in at least five areas of the world. ${ }^{25}$ In addition, infections are associated with several chronic illnesses including juvenile-onset diabetes mellitus, chronic fatigue syndrome, dermatomyositis, polymyositis, congenital hydrocephalus, and amyotrophic lateral sclerosis (ALS). ${ }^{21}$

\section{Treatment}

Treatment of the picornaviruses is primarily symptomatic. While science tries to find new therapeutic modalities, patients have to rely on remedies targeted not to the virus, but to symptoms. Such treatments include firstgeneration antihistamines and nonsteroidal antiinflammatory drugs. Other potential treatments are ipratropium bromide, oral and topical decongestants, antitussives, vitamin C, zinc, and echinacea.

Despite symptomatic therapy, no specific agents are available to treat or prevent enterovirus and rhinovirus diseases. However, characterization of the three-dimensional structure of picornaviruses has allowed development of compounds targeted at the virus itself. On the surface of the virus capsid is a hydrophobic pocket. This pocket is associated with a pore in the canyon floor that accesses a channel leading to the interior of the virus and is believed to have an important role in the virionuncoating process. ${ }^{8}$ The canyon floor is the presumed binding site for ICAM-1 located on epithelial cells. Agents designed to bind to the hydrophobic pocket, known as capsid-binding agents, increase conformational stability of the capsid and thus prevent uncoating of the virus once it enters the intracellular space. Virion uncoating is essential to the life cycle of the virus.

Several capsid-binding agents were developed but did not show promising results clinically. Issues such as complex drug metabolism or lack of broad-spectrum antipicornaviral activity halted their development. Pleconaril (ViroPharma Inc, Exton, PA), a novel, orally available, systemicacting small molecule synthesized in 1991, has antipicornavirus activity and is in clinical trials for treatment of viral respiratory infections.

\section{Pleconaril}

\section{Pharmacology}

Pleconaril exerts its activity on capsid function by integrating with high affinity and specificity in the hydrophobic pocket of the virion. Different steps in the replication process can be affected depending on the specific virus being studied. In the $90 \%$ of rhinoviruses that use ICAM-1 as their cellular receptor, ${ }^{26,27}$ pleconaril works by inhibiting attachment of the virion to the cell, uncoating viral RNA, and producing progeny virions. In all enteroviruses and the $10 \%$ of rhinoviruses that use the low-density lipoprotein receptor, ${ }^{28}$ pleconaril inhibits only the uncoating of viral RNA and production of progeny virions. In either case, the drug prevents the virus from replicating its RNA and thus aborts the infection cycle.

The in vitro antiviral activity of pleconaril was studied against 215 clinical isolates of the most common enterovirus serotypes and 101 rhinovirus serotypes. The drug had potent antiviral activity against 214 enteroviral isolates 
in a concentration range of $0.002-3.4 \mu \mathrm{M}$. The minimum inhibitory concentration of $50 \%$ of isolates $\left(\mathrm{MIC}_{50}\right.$ ) and $\mathrm{MIC}_{90}$ for enteroviral serotypes were $0.03 \mu \mathrm{M}$ or less and $0.18 \mu \mathrm{M}$ or less, respectively. The $\mathrm{MIC}_{50}$ and $\mathrm{MIC}_{80}$ for rhinovirus serotypes were $0.21 \mu \mathrm{M}$ or less and $0.78 \mu \mathrm{M}$ or less, respectively. ${ }^{29}$

\section{Pharmacokinetics}

Pleconaril has linear pharmacokinetics best characterized by a two-compartment open model with first-order absorption. Table 2 shows the drug's pharmacokinetic profile after a single oral dose of 200 or $400 \mathrm{mg}^{30-33}$

The maximum plasma pleconaril concentrations were well above reported MIC $_{50}$ for most nonpolio enteroviral isolates previously tested. ${ }^{34}$ Investigators also found no statistically significant difference in maximum concentration $\left(C_{\max }\right)$ and area under the concentration-time curve (AUC) between men and women and young and elderly subjects, thus concluding that dosage adjustments based on sex and age are not necessary. ${ }^{30}$

In contrast to dose-proportionate pharmacokinetics in adults, increases in $\mathrm{C}_{\max }$ and $\mathrm{AUC}$ were not noted in neonatal patients receiving a single liquid dose of 5.0 or $7.5 \mathrm{mg} / \mathrm{kg}^{34}$ The time to $C_{\max }\left(T_{\max }\right)$ was similar to that of adults $(\sim 3.0$ $\mathrm{hrs}$ ). It is probable that developmental differences in gastrointestinal physiology (i.e., decreased gastric lipolytic activity) affected the extent, but not the rate, of intestinal translocation. Despite the variability, plasma concentrations at 12 hours were adequate for treatment of enteroviral infections in neonates. In children aged 2-12 years receiving a single liquid dose of pleconaril $5 \mathrm{mg} / \mathrm{kg}, \mathrm{C}_{\max }$ and AUC were significantly lower, whereas $T_{\max }$ remained similar to that of adults. ${ }^{35}$ The differences were attributed to increased clearance and volume of distribution in the pediatric population. Nevertheless, plasma pleconaril concentrations 12 hours after the single dose remained approximately 3.5-fold greater than that required to inhibit $90 \%$ of nonpolio enteroviruses in culture.

As mentioned, pleconaril demonstrates firstorder absorption after oral administration. ${ }^{31}$ Its oral bioavailability is approximately $70 \%$ when administered in a fed state. ${ }^{32}$

Animal studies using radiolabeled $\left[{ }^{14} \mathrm{C}\right]$ pleconaril demonstrated peak concentrations in the liver, nasal epithelium, brain, and plasma of
Table 2. Pharmacokinetics of a Single Oral Dose of Pleconaril in Adults ${ }^{30-33}$

\begin{tabular}{|c|c|}
\hline Variable & Value \\
\hline $\begin{array}{c}\mathrm{C}_{\max }(\mathrm{mg} / \mathrm{L})^{\mathrm{a}} \\
200 \mathrm{mg} \\
400 \mathrm{mg}\end{array}$ & $\begin{array}{r}1.1 \pm 0.47 \\
2.17 \pm 0.46\end{array}$ \\
\hline $\begin{array}{l}\text { AUC }(\mathrm{mg} \cdot \mathrm{hr} / \mathrm{L})^{\mathrm{a}} \\
200 \mathrm{mg} \\
400 \mathrm{mg}\end{array}$ & $\begin{array}{l}22.6 \pm 7.47) \\
30.8 \pm 10.9^{b}\end{array}$ \\
\hline $\begin{array}{c}\mathrm{T}_{\max }(\mathrm{hrs})^{\mathrm{a}} \\
200 \mathrm{mg} \\
400 \mathrm{mg}\end{array}$ & $\begin{array}{l}6.0 \pm 4.0 \\
2.5 \pm 2.4^{b}\end{array}$ \\
\hline $\begin{array}{r}V_{d} / F(L)^{a} \\
200 \mathrm{mg} \\
400 \mathrm{mg}\end{array}$ & $\begin{aligned} 1762 & \pm 498 \\
3122-4374 & \pm 1154-1708\end{aligned}$ \\
\hline Bioavailability (\%) & 70 \\
\hline Protein binding (\%) & $>99$ \\
\hline M etabolism & Hepatic \\
\hline Elimination & $\begin{array}{l}<1 \% \text { excreted } \\
\text { unchanged in urine }\end{array}$ \\
\hline $\begin{array}{c}\text { Half-life (hrs) } \\
400 \mathrm{mg} \\
\alpha \text {-Phase } \\
\text { Terminal }\end{array}$ & $\begin{array}{l}\sim 2-3 \\
\sim 180\end{array}$ \\
\hline $\begin{array}{l}\mathrm{C}_{\max }=\text { maximum conc } \\
\text { tration-time curve; } \mathrm{T}_{\text {max }} \\
\text { distribution. } \\
{ }^{\text {a }} \text { Data are mean } \pm \mathrm{SD} \text {. } \\
{ }^{b} \text { M ean value obtained fr }\end{array}$ & 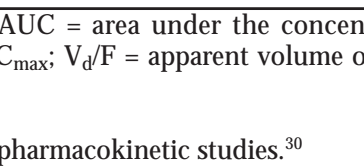 \\
\hline
\end{tabular}

$6.1-17.5,4.2,2.8$, and $0.7 \mathrm{mg} / \mathrm{L}$, respectively, after oral administration. This suggests that the drug penetrates tissue where viral replication is likely to occur at concentrations several times in excess of those in plasma. ${ }^{36}$ Human pharmacokinetic studies involving a single oral dose of 200 or $400 \mathrm{mg}$ determined that despite a protein binding of greater than $99 \%$, the apparent volume of distribution was consistent with significant tissue distribution. ${ }^{33}$

Compared with earlier compounds in this class, a trifluoromethyl substitution on the oxadiazole ring was added to confer greater stability to the molecule against hepatic metabolism. After administration of a single oral dose of pleconaril $200 \mathrm{mg}$ to healthy men, unchanged drug was the major component in plasma for up to 12 hours. ${ }^{37}$ The authors concluded that pleconaril was slowly but extensively metabolized by reductive cleavage of the trifluoromethyl oxadiazole ring to yield amidine derivatives. Further metabolism involved oxygenation and conjunction of the amidine group or oxidation of the isoxazole ring with subsequent ring opening. 
The potential implications of hepatic metabolism on drug interactions were studied in two investigations. ${ }^{37,38}$ In a study of the effect of pleconaril on hepatic levels of cytochrome P450 (CYP) enzymes on rat and dog liver microsomes, the drug had no significant effect on the activity of the major inducible CYP isoforms. ${ }^{39}$ Another study evaluated the effect of pleconaril $400 \mathrm{mg} 3$ times/day for 5 days on the pharmacokinetics of theophylline (as a CYP1A2 enzyme probe) in healthy adults. ${ }^{38}$ Coadministration of the agents resulted in a slight reduction of theophylline's oral clearance and a small increase in its elimination half-life. These changes were not considered clinically significant, and it was concluded that theophylline does not require a dosage adjustment when administered with pleconaril. The authors further concluded that clinically significant pharmacokinetic interactions of pleconaril on other CYP1A2 substrates are unlikely. ${ }^{38}$

Pleconaril was found to exhibit biexponential disposition after administration of single and multiple doses. Both a short $\alpha$-phase half-life ( $\sim 2-3 \mathrm{hrs})$ and long terminal half-life $(\sim 180$ hrs) contributed substantially to elimination. ${ }^{31,37}$ Investigators also found that renal clearance contributes minimally to total systemic clearance of pleconaril, with less than $1 \%$ of the dose excreted unchanged in urine. ${ }^{31}$

\section{Clinical Efficacy and Safety}

Viral Respiratory Infections

A randomized, double-blind, parallel-group, single-site, phase I study evaluated the clinical activity of pleconaril in an experimentally induced coxsackievirus $\mathrm{A} 21$ respiratory infection. ${ }^{40}$ of 33 adult volunteers, 16 were randomized to receive pleconaril administered as a single 400-mg dose on day 1, followed by 200 mg twice/day for 7 days, and 17 were randomized to matching placebo. After the second dose was administered, subjects were inoculated intranasally with 100 plaque-forming units of coxsackievirus A21. Primary outcome measurements were viral shedding, nasal mucus production, respiratory symptom and global illness assessments, antibody titers, pleconaril plasma concentration, and safety. Subjects receiving pleconaril had significantly lower geometric mean viral titers on study days 3,4 , and 7 compared with those given placebo $(p<0.001, p<0.001$, and $p<0.05$, respectively). They also had had significantly less nasal mucus $(p=0.016)$, significantly lower total respiratory symptom scores $(p=0.015)$, and a trend toward reporting less severe disease compared with placebo-treated subjects. Of 31 subjects who had neutralization antibody titers of $1: 4$ or less at baseline, 29 (94\%) seroconverted by study day 28. Minimum plasma concentrations on days 2 and $7(440 \mathrm{ng} / \mathrm{ml}$ and $521 \mathrm{ng} / \mathrm{ml}$, respectively) were several times higher than the concentration necessary to inhibit $50 \%$ of isolates ( $40 \mathrm{ng} / \mathrm{ml}$ ) of the strain of coxsackievirus A21 used in the study. Finally, the frequency of adverse events was similar between groups. ${ }^{39}$

Although that study had a number of strengths, including a gold standard design, assessment of compliance, standardization of routine and concomitant drugs, and an objective safety evaluation, certain limitations must be examined. The study had a small sample size (33 subjects), of whom 1 subject in the placebo group had signs and symptoms of an upper respiratory infection at baseline, 1 had a neutralization titer of 1:4 or greater at baseline, and 2 of 16 receiving pleconaril did not seroconvert. The last limitation perhaps could indicate that these two patients were not infected. Nonetheless, all of these factors could affect the outcomes and provide an advantage to the pleconaril-treated group.

Two randomized, double-blind, placebocontrolled, multicenter, phase II trials evaluated the efficacy and tolerability of pleconaril in the treatment of picornavirus-associated viral respiratory infections (VRI) in adolescents and adults. ${ }^{40}$ Patients aged 14 years or older who came to an outpatient facility within 36 hours of infection onset with an acute, moderate-to-severe respiratory illness (VRI score $\geq 7$ ) and at least one respiratory and one systemic symptom were studied. In the first trial, subjects were randomized to receive a liquid formulation of pleconaril $400 \mathrm{mg} 2$ or 3 times/day, or matching placebo for 7 days. In the second trial, subjects were randomized to receive a tablet formulation of pleconaril $400 \mathrm{mg} 3$ times/day for 7 days. The tablet formulation was chosen because of the increased frequency of gastrointestinal side effects related to the liquid formulation in the first trial. The primary end point of the first trial was time to complete resolution of the six specified symptoms (rhinorrhea, nasal congestion, cough, sore throat, malaise, myalgia), defined as the first of 2 consecutive study days with a total VRI score of zero and no relapse. The primary end point in the second trial was 
time to alleviation of illness, defined as the time until rhinorrhea was absent, and scoring of other symptoms as mild or absent $(\leq 1)$ for at least 48 hours. Other secondary analyses and end points also were evaluated.

The intent-to-treat population consisted of 1015 subjects in the first trial and 875 in the second trial, of whom $41 \%$ and $43 \%$, respectively, had a documented picornaviral infection. The relatively low rate of proven picornaviral illness led investigators to combine data from the two trials to increase the power to assess therapeutic effects. In the intent-to-treat analysis in the first trial, pleconaril 3 times/day was associated with at least a 3-day reduction in the time to complete resolution of all symptoms compared with placebo ( 11.0 vs $>14.0$ days, $p=0.010$ ), whereas the twice-daily regimen showed no difference with the placebo group. Furthermore, the time to illness alleviation for patients receiving pleconaril 3 times/day was 1 day earlier than that in the placebo group ( 7.5 vs 8.5 days, $p=0.006$ ), with no difference between the twice-daily regimen and placebo. As a result, efficacy analyses focused on a comparison of pleconaril 3 times/day and matching placebo in patients with documented picornavirus infection (pleconaril 323 patients, placebo 264).

Time to illness alleviation was significantly shorter in pleconaril-treated patients ( 8.5 vs 10.0 days, $p=0.029$ ). Time to complete resolution of symptoms favored pleconaril and showed a trend toward a significant difference ( 11.5 vs 12.5 days, $p=0.177)$. The time to alleviation of rhinorrhea and all other VRI symptoms and time to reduction of $50 \%$ or more from baseline in total symptom severity score was statistically different and favored pleconaril in both analyses (10.0 vs 8.5 days, $p=0.029 ; 4.5$ vs 3.5 days, $p=0.038$ ). The self-reported time to overall resolution of illness was 1 day shorter in the pleconaril group (10.5 vs 11.5 days, $p=0.07)$. Results of secondary end point measures also favored pleconaril: the sum of total symptom severity scores was 13\% lower $(p=0.007)$, the number of tissues used was reduced by $20 \%$ through day $14(p=0.018)$, and the proportion of nights with sleep disturbances was $16 \%$ lower in the pleconaril group $(p=0.053)$.

The safety profile of pleconaril was evaluated for all subjects in the two trials. ${ }^{40}$ The first trial revealed no statistically significant difference in adverse-event profiles between pleconaril and placebo. The most common adverse events were diarrhea, nausea, abdominal pain, and headache.
The use of a tablet formulation in the second trial reduced the overall frequency of gastrointestinal side effects but did have a higher frequency of nausea compared with placebo $(p=0.003)$. Patients in the pleconaril group had an increase in serum total cholesterol compared with placebo (median change from baseline $+8 \mathrm{mg} / \mathrm{dl}$ and no change, respectively, $p<0.001$ ). The investigators concluded that oral pleconaril 3 times/day was generally well tolerated and reduced the duration and severity of picornaviral respiratory infection in adolescents and adults.

Limitations of this study must be examined and include the subjectivity of the primary end points. Different patient perceptions of illness and its severity can influence outcome. Furthermore, use of prescription and over-thecounter agents was not restricted. It is interesting to note that patients who used concomitant cold symptom-relief drugs showed less benefit from pleconaril than those who did not. This could be related once again to subjects' perception of illness, need for alternative therapy, and reporting of symptoms and their resolution.

Two randomized, double-blind, placebocontrolled, multicenter, phase III trials evaluated the efficacy and safety of pleconaril for the treatment of VRIs in subjects aged 18 years or older with self-diagnosed colds of 24 hours' duration or less. ${ }^{41}$ The two trials were of identical design and were conducted concurrently at different centers in the United States and Canada. Patients were randomized to receive either pleconaril $400 \mathrm{mg} 3$ times/day for 5 days or matching placebo. The primary efficacy end point was time to alleviation of illness, defined as resolution of rhinorrhea (VRI score 0 ), mild to absent on all other VRI symptoms (score $\leq 1)$, and 48 hours or more without taking symptom-relief agents. Of 2096 patients randomized, $65 \%$ had a documented picornaviral infection and became the primary study population for evaluating efficacy (pleconaril 681, placebo 682). Compared with placebo recipients, pleconaril-treated subjects had a significantly shorter time to alleviation of illness ( 4.3 vs 6.3 days, $p<0.001$ ), shorter time to alleviation of illness regardless of documented picornavirus infection $(p=0.009)$, and shorter time to resolution of individual symptoms. The median percentage reduction in total VRI symptom severity score was significantly lower in the pleconaril group by the morning of the second day and remained significantly lower until complete resolution of infection $(p=0.05$ 
and $p<0.001$, respectively). The percentage of patients with any bothersome symptoms was also significantly lower in the pleconaril-treated group $(p<0.05)$.

The safety evaluation included all patients randomized in the trials (pleconaril 1046, placebo 1050). There was no statistical difference in adverse events between the pleconaril and placebo groups. The only notable laboratory finding associated with pleconaril was an increase from baseline in serum total cholesterol (median change from baseline of $+5 \mathrm{mg} / \mathrm{dl}$ for pleconaril, $-4 \mathrm{mg} / \mathrm{dl}$ for placebo, $p<0.001$ ), which was considered to be of clinical significance. It was concluded that pleconaril $400 \mathrm{mg} 3$ times/day for 5 days reduced the severity of common cold symptoms as early as the second day of treatment, reduced the duration of illness, and was associated with an excellent safety profile.

\section{Enteroviral Meningitis}

A double-blind, placebo-controlled, multicenter trial was conducted in 130 patients aged 14-65 years with polymerase chain reaction (PCR)confirmed enteroviral meningitis in whom severe headache was present for less than 48 hours. ${ }^{42}$ Patients were given pleconaril $200 \mathrm{mg} 3$ times/day for 7 days or matching placebo. Resolution of headache and other meningitis symptoms was 2 days shorter in the pleconariltreated group $(p=0.04)$. Pleconaril-treated patients also returned to work or school 2 days earlier than placebo-treated patients $(p=0.045)$. There was no statistically significant difference in adverse events between groups.

A double-blind, placebo-controlled, international study evaluated children aged 4-14 years with headache, at least one other symptom of viral meningitis, and the presence of more than 5 white blood cells in cerebrospinal fluid. ${ }^{43}$ Patients were randomized, within 48 hours after onset of symptoms to one of three treatments: placebo, pleconaril $2.5 \mathrm{mg} / \mathrm{kg} 3$ times/day for 7 days, or pleconaril $5 \mathrm{mg} / \mathrm{kg} 3$ times/day for 7 days. Primary end points were time to absence of headache, absence of systemic symptoms as measured by a global assessment score (GAS), and a multidimensional total morbidity score (TMS).

Of 221 patients randomized, 181 (82\%) had confirmed enteroviral infection. Pleconaril 2.5 $\mathrm{mg} / \mathrm{kg} 3$ times/day for 7 days reduced the percentage of subjects with persistent headache and TMS and GAS scores above zero at the conclusion of treatment by $50 \%, 38 \%$, and $46 \%$, respectively, compared with placebo. The response to low-dose pleconaril was noted as early as 24 hours after starting therapy. The low dosage also reduced the median duration of elevated TMS and GAS scores from 7 and 8 days, respectively, in the placebo group to 6 days in the treatment group ( $p=0.03, p=0.05$ ). Duration of headache was reduced by 1 day by low-dose pleconaril for patients aged 8 years or older ( 5 vs 6 days, $p=0.075$ ).

In the high-dose pleconaril group, the percentage of patients reporting headache was reduced at first but was not sustained. Viral shedding on days 4 and 8 was reduced with both the low- and high-dose pleconaril regimens. The investigators found no statistically significant differences between the pleconaril and placebo groups.

\section{Other Enteroviral Infections}

Preliminary outcomes of potentially lifethreatening enteroviral infections treated with the compassionate-use pleconaril protocol over 2.5 years were reported. ${ }^{44}$ Eligible patients were those with chronic enteroviral meningoencephalitis and agammaglobulinemia or hypogammaglobulinemia (CEMA), neonatal enterovirus sepsis, myocarditis, vaccineassociated or wild-type polioviral infection, postpolio muscular atrophy syndrome, or enteroviral encephalitis, and bone marrow transplant (BMT) recipients who were infected with enterovirus. Dosages were $5.0 \mathrm{mg} / \mathrm{kg} 3$ times/day for 7-10 days for children and 200-400 mg 3 times/day for 7-10 days for adults. Response to therapy was defined as follows: clinical response-diminution of one or more prominent presenting signs or symptoms; virologic response- reversion from enterovirus culture-positive to culture-negative and/or from enterovirus PCR-positive to PCR-negative; laboratory response-improvement or return to normal of abnormal laboratory characteristics specific to the disease; radiologic responseobjective improvement in findings of either magnetic resonance imaging or technetium $99 \mathrm{~m}$ bisicate brain scans, or chest radiographs. Results were presented based on the number of patients who had enough data for each parameter.

Of 17 patients with CEMA, 12/16 had clinical, 6/7 virologic, 8/9 laboratory, and 3/3 radiologic 
improvement. Of six neonatal patients treated for overwhelming enteroviral sepsis, 5/6, 4/4, and $4 / 5$ had clinical, virologic, and laboratory responses, respectively. There were three patients with enterovirus and one patient with rhinovirus BMT-related infection. Of three BMT recipients with enterovirus infection, 2/2 had clinical and $1 / 1$ had a laboratory response. The patient with rhinovirus had a clinical but not a radiologic response. Three patients were treated for acute poliovirus infections ( 2 vaccine associated, 1 wild-type). Of the three patients, 2/3 had clinical, $1 / 1$ had virologic, and $1 / 1$ had laboratory responses. One patient with ALS was treated with pleconaril and had a clinical response. This patient was included due to reports associating enteroviral infection and ALS. ${ }^{45}$ One patient with acute onset of enteroviral transverse myelitis and encephalitis had both neurologic and respiratory improvements related to pleconaril. Of two patients treated for enteroviral encephalitis, one improved clinically and the other remained unchanged after therapy. These results suggest that the drug has a beneficial effect on clinical, virologic, laboratory, and radiologic values in patients with potentially life-threatening enteroviral infections.

\section{Place in Therapy}

Pleconaril is a novel, orally available and systemically acting molecule that exhibits potent antiviral activity against rhinovirus serotypes associated with the common cold. Pleconaril 400 mg 3 times/day for 5-7 days given within 24-36 hours of symptom onset decreases the duration and severity of the common cold in nonsmoking patients with documented picornaviral respiratory tract infections, and has an adverseevent profile similar to that of placebo. In the United States alone, there are over 1 billion colds annually, ${ }^{1}$ of which the rhinoviruses are the leading cause. Furthermore, rhinoviruses are a predominant cause of asthmatic exacerbations ${ }^{2,3}$ and severe lower respiratory tract disease. Pleconaril is the first antipicornaviral agent to demonstrate activity in large clinical trials; however, its place in therapy against these viruses remains to be determined since it did not receive Food and Drug Administration (FDA) approval for use in adult patients with viral respiratory infections.

Issues of adverse effects, drug interactions, and resistance are of greatest concern to the FDA. In pivotal trials, adverse effects with greater frequency than placebo were gastrointestinal events and headache. However, studies evaluating a longer duration of therapy, particularly the 6-week prophylactic study, revealed a more concerning safety profile. In these studies menstrual disorders such as early menses, breakthrough bleeding, and menorrhagia, occurred in 3-81\% of women taking oral contraceptives. An increased frequency was observed with a longer duration of pleconaril use and with twice-daily dosing. These events are thought to be the result of CYP3A4 induction. Results from a follow-up drug interaction study in fact reported decreases in estradiol levels by $35 \%$. The potential for unintended pregnancies in women taking pleconaril and oral contraceptives is therefore a major concern. Among oral contraceptive users, unintended pregnancies occurred in two women taking pleconaril and in one taking placebo.

Tachycardia with or without palpitations was reported by seven pleconaril-treated patients. Although none of them was treated concomitantly with theophylline, this finding prompted a theophylline interaction study. Conducted in 15 healthy volunteers, it reported a $15 \%$ increase in the theophylline AUC together with increased frequencies of abdominal pain, nausea, dizziness, and syncope. Three patients experienced palpitations, although they showed no significant pharmacokinetic changes. There are no data on other compounds that may be affected by this enzyme system, such as immunosuppressants, antiarrhythmics, calcium channel blockers, and protease inhibitors. ${ }^{46}$

Issues related to resistance are also of concern. Approximately $24 \%$ of patients had resistance to pleconaril. Thirteen percent had resistance at baseline, without previous exposure to the drug. Another $11 \%$ developed resistance during therapy. Molecular analysis of the resistance trends showed that single amino acid substitutions could result in up to an 100-fold decrease in susceptibility. ${ }^{4}$

In addition, the effects pleconaril may have on different subgroups are of interest. Subjects enrolled in pivotal studies were healthy adults, predominantly white, and $70 \%$ women. Since pleconaril may be beneficial for patients with severe colds who have complications or are at risk of developing complications from their infections, the FDA recommended that a broader population be studied, to include asthmatics, children, elderly, and immunosuppressed; those with chronic cardiac or pulmonary disease; and 
those from other ethnic backgrounds such as African-Americans and Hispanics. ${ }^{4}$ An important analysis revealed that pleconaril did not have any beneficial effect on patients who smoke.

Although pleconaril was not submitted to the FDA for approval of enteroviral meningitis or other life-threatening enteroviral infections, it should be considered as possible first-line therapy through the company's compassionate use program. Enteroviruses are the most common cause of meningitis in the United States and an important cause of encephalitis, poliomyelitis, myocarditis, hemorrhagic conjunctivitis, hand-foot-mouth syndrome, pleurodynia, and nonspecific febrile illnesses. ${ }^{4,5}$ Pleconaril administered within 48 hours of symptom onset at stated dosages decreased the duration and severity of enteroviral meningitis and offered other improvement in patients with other severe enteroviral infections. It is clear that additional studies are required to evaluate fully the risk-benefit potential of pleconaril before wide-scale clinical administration can be advocated.

\section{References}

1. National Institute of Allergy and Infectious Diseases. Common cold fact sheet. Bethesda, MD: National Institute of Health, 1998.

2. Halonen P, Rocha E, Hierholzer J, et al. Detection of enteroviruses and rhinoviruses in clinical specimens by PCR and liquid-phase hybridization. J Clin Microbiol 1995;33(3):648-53.

3. Chidekel AS, Rosen CL, Buzzy AR. Rhinovirus infection associated with serious lower respiratory illness in patients with bronchopulmonary dysplasia. Pediatr Infect Dis J 1997;16(1):43-7.

4. Rotbart HA. Meningitis and encephalitis. In: Rotbart HA, ed. Human enterovirus infections. Washington, DC: American Society for Microbiology Press, 1995:271-89.

5. Whitley RJ, Cobbs CG, Alford CA, et al. Diseases that mimic herpes simplex encephalitis. JAM A 1989;262:234-9.

6. Strikas RA, Anderson LJ, Parker RA. Temporal and geographic patterns of isolates of nonpolio enterovirus in the United States, 1970-1983. J Infect Dis 1986;153:346-51.

7. Treanor J. Respiratory infections. In: Richman DD, Whitley RJ, Hayden FG, eds. Clinical virology. New York: Churchill Livingstone, 1997:5-33.

8. Gwaltney JM. Rhinovirus. In: Mandell GL, Bennet JE, Dolin R, eds. Principles and practice of infectious diseases, 5th ed. New York: Churchill Livingstone, 2000:1940-8.

9. Monto AS, Cavallaro JJ. The Tecumseh study of respiratory illness. II. Patterns of occurrence of infection with respiratory pathogens, 1965-1969. Am J Epidemiol 1971;94:280-9.

10. van Kempen $\mathbf{M}$, Bachert $\mathbf{C}$, Van $\mathbf{C}$ auwenberge $\mathbf{P}$. An update on the pathophysiology of rhinovirus upper respiratory tract infections. Rhinology 1999;37:97-103.

11. Winther B, G waltney JM Jr, Mygind N, et al. Sites of recovery after point inoculation of the upper airway. JAMA 1986;256:1763-7.

12. Turner RB. The treatment of rhinovirus infections: progress and potential. Antiviral Res 2001;49:1-14.

13. Arola $\mathbf{M}$, Ruuskanen $\mathbf{0}$, Ziegler $\mathbf{T}$, et al. Clinical role of respiratory virus infection in acute otitis media. Pediatrics
1990;86:848-55

14. Nicholson KG, Kent J, Hammersley V, et al. Risk factors for lower respiratory complications of rhinovirus infections in elderly people living in the community: prospective cohort study. BMJ 1996;313:1119-23.

15. Wald TG, Shult P, Krause P, et al. A rhinovirus outbreak among residents of a long-term care facility. Ann Intern Med 1995;123:588-93.

16. Schmidt HJ, Fink RJ. Rhinovirus as a lower respiratory pathogen in infants. Pediatr Infect Dis J 1991;10:700-2.

17. Chidekel AS, Bazzy AR, Rosen CL. Rhinovirus infection associated with severe lower respiratory tract illness and worsening lung disease in infants with bronchopulmonary dysplasia. Pediatr Pulmonol 1994;18:261-3.

18. Nicholson KG, Kent J, Ireland DC. Respiratory viruses and exacerbations of asthma in adults. BMJ 1993;307:982-6.

19. Johnston SL, Pattermore PK, Sanderson G, et al. Community study of role of viral infections in exacerbations of asthma in 9-11 year old children. BMJ 1995;310:1225-9.

20. Johnston SL, Pattermore PK, Sanderson G, et al. The relationship between upper respiratory infections and hospital admissions for asthma: a time-trend analysis. Am J Respir Crit Care Med 1996;154:654-60.

21. Minor TE, Baker JW, Dick E, et al. Greater frequency of viral respiratory infection in asthmatic children as compared with their nonasthmatic siblings. Pediatrics 1974;85:472-7.

22. Stenhouse AC. Rhinovirus infection in acute exacerbations of chronic bronchitis: a controlled prospective study. $\mathrm{Br}$ Med J 1967;3:461-3.

23. Cherry JD. Enteroviruses: coxsackieviruses, enteroviruses and polioviruses. In: Feigin RD, Cherry JD, eds. Textbook of pediatric infectious diseases, 4th ed. Philadelphia: WB Saunders, 1998:1787-1839.

24. Wu JM, Wang JN, Tsai YC, et al. Cardiopulmonary manifestations of fulminant enterovirus 71 infection. Pediatrics 2002;109:1-5.

25. Lin TY, Chang LY, Hsia SH, et al. The 1998 enterovirus 71 outbreak in Taiwan: pathogenesis and management. Clin Infect Dis 2000;34(suppl):S52-7.

26. Greve JM, Davis G, Meyer AM, et al. The major rhinovirus receptor is ICAM-1. Cell 1989;56:839-47.

27. Staunton DE, Merluzzi VJ, Rothlein R, et al. A cell adhesion molecule, ICAM-1 is the major surface receptor for rhinoviruses. Cell 1989;56:849-53.

28. Hofer $\mathbf{F}, \mathbf{G r u e n b e r g e r} \mathbf{M}$, Kowlaski $\mathbf{H}$, et al. Members of the low density lipoprotein receptor family mediate cell entry of a minor-group common cold virus. Proc Natl Acad Sci USA 1994;91:1839-42.

29. Pevear DC, Seipel ME, Pallansch M, et al. In vitro activity of VP 63843 against field isolates of non-polio enteroviruses [abstr]. In: Program and abstracts of the 36th interscience conference on antimicrobial agents and chemotherapy. Washington, DC: American Society for Microbiology, 1996:181.

30. Rhodes G, Liu S. Effects of age and gender on pleconaril pharmacokinetics [abstr]. In: Program and abstracts of the 41st interscience conference on antimicrobial agents and chemotherapy. Washington, DC: American Society for Microbiology, 2001:12.

31. Rhodes G, Liu S. Multiple dose pharmacokinetics of pleconaril tablets in healthy adults [abstr]. In: Program and abstracts of the 41st interscience conference on antimicrobial agents and chemotherapy. Washington, DC: American Society for Microbiology, 2001:12.

32. Abdel-Rahman SM, Kearns GL. Single dose pharmacokinetics of pleconaril (VP 63843) oral suspension and effect of food. Antimicrob Agents Chemother 1998;42:2706-9.

33. Hincks J, Rhodes G, Liu S. Pharmacokinetics and metabolism of $\left[{ }^{14} \mathrm{C}\right]$-pleconaril in healthy adult males [abstr]. In: Program and abstracts of the 41 st interscience conference on antimicrobial agents and chemotherapy. Washington, DC: American Society for Microbiology, 2001:12.

34. Kearns GL, Bradley JS, Jacobs RF, et al. Single dose pharmacokinetics of pleconaril in neonates. Pediatr Infect Dis J 
2000;19:833-9.

35. Kearns GL, Abdel-Rahman SM, James LP, et al. Single dose pharmacokinetics of pleconaril (VP63843) oral solution in children and adolescents. Antimicrob Agents Chemother 1999;42(10):634-8.

36. Viropharma, Inc. VP 63843 clinical investigator's brochure. Exton, PA; 2001

37. Rhodes G, Haughey D, Liu S. Effect of repeat doses of pleconaril on pharmacokinetics of theophylline in healthy adults [abstr]. In: Program and abstracts of the 41st interscience conference on antimicrobial agents and chemotherapy. Washington, DC: American Society for Microbiology, 2001:13.

38. Rhodes G, Harling S, Hincks J. Effect of pleconaril on hepatic levels of CYP450 enzymes in rat and dog liver microsomes following in vivo dosing [abstr]. In: Program and abstracts of the 41st interscience conference on antimicrobial agents and chemotherapy. Washington, DC: American Society for Microbiology, 2001:13.

39. Schiff GM, Sherwood JR. Clinical activity of pleconaril in an experimentally induced coxsackievirus A21 respiratory infection. J Infect Dis 2000;181:20-6.

40. Hayden FG, Coats T, Kim K, et al. Oral pleconaril treatment of picornavirus-associated viral respiratory illness in adults: efficacy and tolerability in phase II clinical trials. Antiviral Ther 2002;7:53-65.
41. Hayden FG, Kim K, Hudson S. Pleconaril treatment reduces duration and severity of viral respiratory infection (common cold) due to picornaviruses [abstr]. In: Program and abstracts of the 4lst interscience conference on antimicrobial agents and chemotherapy. Washington, DC: American Society for Microbiology, 2001:288.

42. Shafran SD, Halota W, Gilbert D, et al. Pleconaril is effective for enteroviral meningitis in adolescents and adults: a randomized placebo-controlled multicenter trial [abstr]. In: Program and abstracts of the 39th interscience conference on antimicrobial agents and chemotherapy. Washington, DC: American Society for Microbiology, 1999:436.

43. Sawyer MH, Saez-Llorenz X, Aviles CL, et al. Oral pleconaril reduces the duration and severity of enteroviral meningitis in children. Presented at the annual meeting of the Pediatric Academic Society, San Francisco, CA, May 1-4, 1999.

44. Rotbart HA, Webster AD. Treatment of potentially lifethreatening enterovirus infections with pleconaril. Clin Infect Dis 2001;32:228-35.

45. Berger MM, Kopp N, Vital $\mathbf{C}$, et al. Detection and cellular localization of enterovirus RNA sequences in spinal cord of patients with ALS. Neurology 2000;54:20-5.

46. Food and Drug Administration. Antiviral drugs advisory committee report. Available from www.fda.gov/ohrms/dockets/ ac/02/transcripts/3847t1.pdf. Accessed N ovember 10, 2002. 